\title{
Influence Maximization with Consideration of PageRank Centrality and Propagation Probability
}

\author{
Qi Chen,Rong Yan* \\ Inner Mongolia Key Laboratory of Mongolian Information Processing Technology \\ College of Computer Science, Inner Mongolia University \\ Hohhot, China \\ Email: 1254560848@qq.com,csyanr@imu.edu.cn
}

\begin{abstract}
Influence Maximization (IM) problem has been attracted considerable interest and attention in last decades. However, the centrality algorithm-based methods were with low time complexity but made the acceptability of diffusion vaguely. The main purpose of our work is to select the influential nodes according to the available budget to maximize the impact coverage. Based on the traditional independent cascade model, this paper mainly solves the IM problem, designs two effective PRTH algorithms based on PageRank and propagation probability threshold, and combines PageRank of PRTH processed graph with degree discount algorithm to get an algorithm named PRDD. Experiments on four datasets show that the two algorithms have better performance than the existing algorithms in the aspect of influence diffusion.
\end{abstract}

Index Terms-influence maximization, PageRank, degree discount, propagation probability

\section{INTRODUCTION}

Identifying an initial seed set of users who would eventually affect the maximum number of users is the Influence Maximization (IM) problem. It is of great significance in controlling public opinion, rumors and advertising marketing, and it has been become a hot research field due to the increase in the number and scale of interaction between users in social networks. At the same time, it also brings massive data to the network, and the value of network data is also increasing. IM was first used in marketing, which is called viral marketing. The spread pattern of viral marketing [1] is based on mutual trust between two users. The purpose of viral marketing is to influence their friends, promote products or pass information to their friends, and then expend this scheme maximization as much as possible, as well as the coverage of information. One of the most active areas of interest in the last decade has been impact maximization and how content or products are adopted by users, which was first introduced in the work of Kempe et al. [2]. Then, in view of the challenge of this problem, kinds of research work have been carried out. Kempe et al. [2] considered IM problem as a combinatorial problem, and proposed an effective greedy algorithm, which can achieve high influence spread among all existing methods, but losing in time complexity. Great efforts followed the work of Kempe et al. [2] and tried to reduce the running time, such as CELF [3] and CELF++ [4]. However, few greedy algorithmbased methods are feasible for large-scale network, while the heuristic method, such as degree discount, instead can achieve an acceptable impact in running time [5].

At present, the existing centrality algorithm-based methods merely use the node degree as a measurement, but ignore the node PageRank. In this paper, we devise a new top$k$ selection algorithm based on PageRank centrality and propagation probability threshold (PRTH). We calculate The influence diffusion and marginal gain by successive iterative updating. Furthermore, we propose a new PRDD algorithm by combining PRTH with degree discount algorithm to alleviate the effect of the point aggregation. These above algorithms make it possible to calculate the influence diffusion directly and accurately. Through experimental analysis, our algorithm has achieved acceptable results, compared with the current mainstream algorithm in terms of influence diffusion.

The remainder of the paper is organized as follows. Section 2 reviews the related work. Section 3 explains the design and implementation of our methods in detail. Experimental results on four large datasets are provided in Section 4. Finally in Section 5 , we conclude this paper.

\section{RELATED WORK}

IM problem was firstly proposed by Domingo et al. [1] from the perspective of algorithm. Next, Kempe et al. [2] formulated the issue of IM in the social network, and proposed two widely influence diffusion models: Linear threshold model (LT) and Independent Cascade model (IC). As for a non-deterministic polynomial-time (NP)-hard problem, Kempe et al. [2] proposed a greedy approximation algorithm with $(1-1 / E)$ approximation ratio to address this issue. Experimental results showed that the proposed algorithm was more effective than the traditional random methods. However, greedy algorithms in large-scale social networks were inefficient and inaccurate with poor time complexity and memory consumption. The reason mainly is that the greedy approximation ratio method requires tens of thousands of Monte Carlo simulations. In recent years, much efforts were proposed to effectively address IM problem. The related research includes the greedy-based methods and the heuristic-based methods.

Pursuing low time complexity is the question surround the greedy-based algorithms. lots of researchers tried to improve the greedy-based methods and provide kinds of effective 
versions. Leskovec et al. [3] proposed CELF algorithm to enhance the greedy algorithm by using sub module characteristics in node selection process. The CELF algorithm could obviously reduce the evaluation times than the simple greedy algorithm. Goyal et al. [4] proposed a modified CELF algorithm, called CELF++, which can significantly reduce the amount of computation and obtain the better results. Chen et al. [5] designed a new scheme to improve the greedy algorithm, and combined their model with CELF algorithm to get a faster greedy algorithm. Furthermore, they proposed the prefix of exclusion maximum impact tree by using local tree model to approximate influence propagation.

Heuristic-based algorithms try to improve the propagation effect, and much heuristic-based literatures are based on centrality. Chen et al. [6] exploited a degree discount heuristic algorithm, which nearly matched the performance of the greedy algorithms for the IC model, and improved upon the pure degree heuristic in the other cascade models. Nandi et al. [7] proposed a new method called DegGreedy to maximize the influence spread based on node neighborhoods, which could provide higher influence spread and good efficiency in terms of scalability. Deng et al. [8] proposed two centrality-based edge activation probability algorithms under the IC model, which named NewDiscount and GreedyCIC, with considering edge probability. Taheri et al. [9] utilized HellRank centrality measure to identify the most influential users based on the Hellinger distance between a node pair in a bipartite graph. Cui et al. [10] proposed a degree-descending search strategy to obtain all nodes that have the influence spread as the degree centrality. Lattanzi et al. [11] designed a random node centrality algorithm based on the phenomenon of friendship paradox. Mohammed et al. [12] proposed a new algorithm called PrKa based on Katz centrality. In their work, the propagation probability threshold permitted to compute the influence over all the paths and selected the one that maximizes the influence. Recently, Ding et al. [13] proposed a new realistic independent cascade (RIC) model and several greedy maximization algorithms. Maji et al. [14] modified the $k$-shell method and compared several variants. Banerjee et al. [15] proposed a hop-based heuristic method based on 'expected earned benefit'.

\section{Methodology}

Firstly, we give some denotes for the work. We consider the social network as a undirected graph $G=(V, E)$, where $V$ denotes a group of users, $E$ denotes a group of relationships. We represent the number of users in the network as $|V|$ and $|E|$ as the number of edges.

\section{A. Algorithm (PRTH): Pagerank centrality and propagation probability threshold algorithm.}

In this paper, we assume that the larger the PageRank value is, the more important the node is, and the information is easy to spread from important nodes to other nodes. But you can imagine such a situation, when a node connects many edge nodes with degree 1, its PageRank value will be very high, but it is not so important. According to the above assumptions, we solve the problem by the following methods. We set a weight for each edge and take the average of ownership as the threshold. Then the edge whose weight is less than the threshold is removed. This method can effectively remove the edge nodes, making the distribution of PageRank points more concentrated in the central area, also for the propagation path of nodes wider.

Next we will introduce our PRTH algorithm. Firstly, we construct a undirected graph. Next, we need to get the PageRank value of each node in the graph. Then, we calculate the edge weight divided by the value of each user with the sum of the PageRank of the two users connected using the edge. Through the above description, we can obtain the node pair weight using equation (1):

$$
\operatorname{weight}(u, v)=\frac{\operatorname{pagerank}(u)}{\operatorname{pagerank}(v)+\operatorname{pagrank}(u)}
$$

Where $u$ and $v$ denote two different nodes, respectively. Through equation (1), we can clearly conclude that the weight of $u$ to $v$ is different from that of $v$ to $u$. So here we choose the maximum weight of each edge pair as the weight of the each edge pair.

With these steps we get a weighted graph. After that, we introduce the threshold of propagation probability, which is reserved only when the weight of the edge reaches this value. The probability is simply calculated by the average of the weight of the edges and the number of edges. Therefore, we obtain the maximum propagation probability threshold $t h$ using the equation (2):

$$
t h=\frac{\sum_{e}^{E} \text { weight }_{\max }(e)}{|E|}
$$

After removing the unqualified edges, we get a new graph. Next, we will calculate the PageRank value in the new graph again and arrange it in descending order afresh. Finally, the top- $k$ node set before sorting are selected as the final seed node set. We use Algorithm 1 to accomplish it.

\section{B. Algorithm (PRDD): Combining pagerank with degree dis-} count.

Previously, we have concluded that PRTH algorithm can make the node distribution closer to the center. Unfortunately, we found that the node aggregation occurs occasionally in the experiments, and PRTH algorithm may become unstable as the number of seed nodes increases. Furthermore, we propose an algorithm named PRDD to modify the situation. In this algorithm, we fuse the index of PRTH and the index of degree discount in the original graph, so as to improve the ability of preventing aggregation.

In this section, we will introduce the influence maximization model combining PageRank and degree discount. First of all, we need to calculate the PRTH and the degree discount of the graph. Secondly, we normalize the two values of PageRank and degree discount, and then combine the two indicators through a linear parameter $\alpha$, which can be computed by 


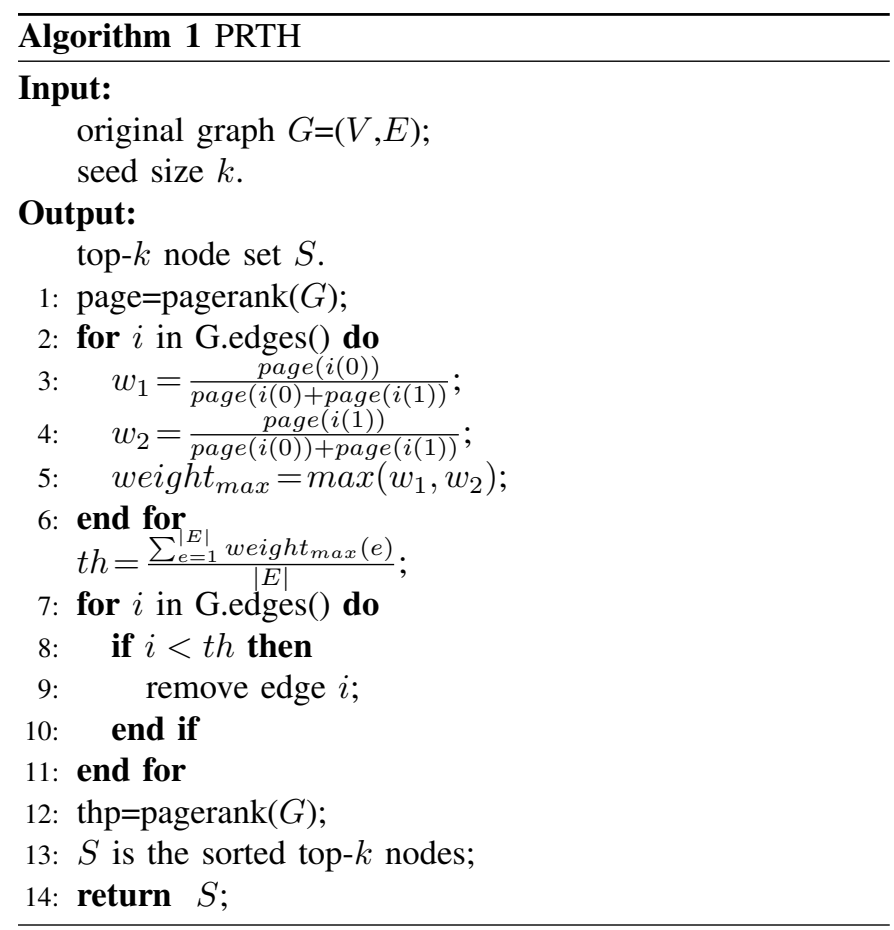

equation (3). The best value of $\alpha$ is from 0.1 to 0.3 in the experiments.

$$
P D(v)=(1-\alpha) * \frac{p(v)}{\text { max_pagerank }}+\alpha * \frac{d d(v)}{\text { max_degree }}
$$

Algorithm 2 gives the specific process for combining PageRank with degree discount algorithm (PRDD).

\section{EXPERIMENTAL RESULTS}

In this section, we conduct our experiments on four undirected graphs. We focus on the performance of the algorithm in terms of running time and influence diffusion.

\section{A. Datasets and Experiment Settings}

First, we introduce the datasets used in the experiments. We use a social network dataset Facebook ${ }^{1}$ and three datasets of academic collaboration network: CA-HepTh ${ }^{2}$, NetHEPT and NetPHY ${ }^{3}$.

1) Facebook: This dataset of 'circles' (or 'friends lists') is from Facebook. The data of Facebook is collected from survey participants.

2) CA-HepTh: Arxiv HEP-TH (High Energy Physics Theory) collaboration network is from the arXiv ${ }^{4}$ and covers scientific collaborations between authors papers submitted to High Energy Physics - Theory category.

3) NetHEPT: It is an academic collaboration network from arXiv. In this dataset two of them are connected by an undirected link, if they co-authored at least one paper.

${ }^{1}$ http://snap.stanford.edu/data/ego-Facebook.html

${ }^{2}$ http://snap.stanford.edu/data/ca-HepTh.html

${ }^{3}$ https://www.microsoft.com/en-us/research/wp-content/uploads/2016/02/weic graphdata.zip

${ }^{4}$ https://arxiv.org/

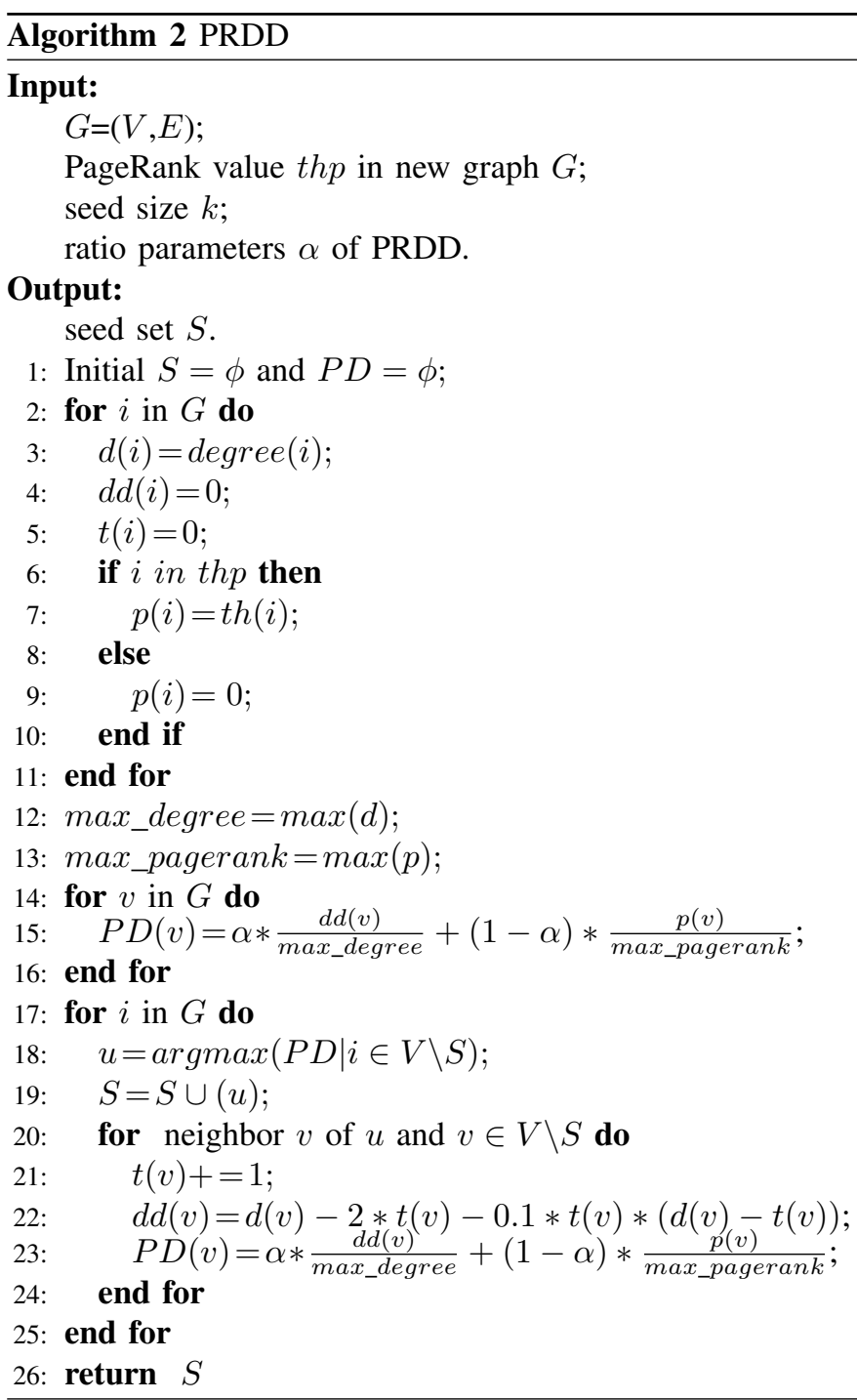

4) NetPHY: It is an academic collaboration network among the researchers. In this dataset two of them are connected by an undirected link, if they co-authored one paper.

Table I gives the detailed description of the datasets.

TABLE I: The description of the datasets.

\begin{tabular}{|c|r|r|r|}
\hline Datasets & Nodes number & Edges number & Avg. degree \\
\hline Facebook & 4,039 & 88,234 & 43.69 \\
\hline CA-HepTh & 9,877 & 25,998 & 5.26 \\
\hline NetHEPT & 15,233 & 58,891 & 7.73 \\
\hline NetPHY & 37,154 & 231,507 & 13.4 \\
\hline
\end{tabular}

As for both above algorithms, we conduct the experiments under the IC model with probability $p$ set to 0.1 . The seed size $k$ varies from 10 to 50 , and the number of iteration is set to 1000 .

\section{B. Baseline}

In the experiments, five baseline algorithms are compared. The descriptions are presented as below. 
1) Degree algorithm: This algorithm selects the degree centrality of the top- $k$ propagator with the highest degree centrality.

2) Pagerank [16] algorithm: This algorithm counts the number and quality of links from a node to all other nodes to determine the importance of the node. We also choose the first $\mathrm{k}$ values as nodes.

3) Degree Discount [6] algorithm: This algorithm selects the seed set according to the degree center degree score of nodes, and discounts the edge combined with the next selected seed from the degree calculation of nodes.

4) PrKatz [12] algorithm: This algorithm is relied on the use of a combination of Katz centrality and propagation probability threshold tested over each edge for each user in the network.Its parameters are the same as those in the article [12] $=1,=0.0015$.

5) RIS [17] algorithm: This algorithm generates reverse reachable set to find the maximum seed nodes.

\section{Evaluation and Analysis}

First of all, we did an experiment to explore the effect of a on the results. We set the value of a from 0.1 to 0.9 , and we did experiments on all data sets. In this paper, we present the experimental results on Facebook and NetPHY datasets. We

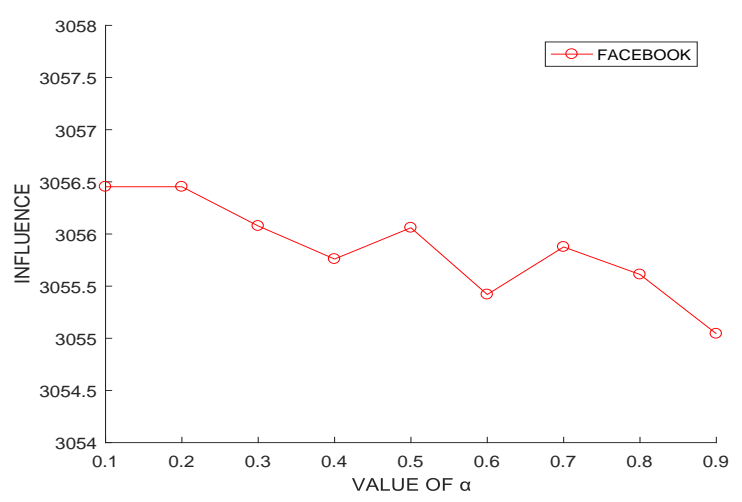

Fig. 1: The result on Facebook.

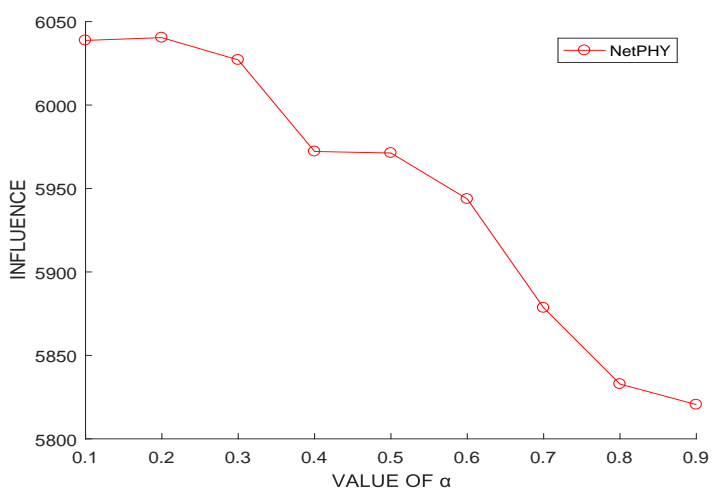

Fig. 2: The result on Facebook. can see that the best a value is between 0.1 and 0.3.Similar conclusions can be obtained on the other two datasets.

Next, we compared the baseline method with four datasets as follows:

Figure. 3 shows the effect of all algorithms in the Facebook dataset. Compared with other datasets, this dataset has much more edges.

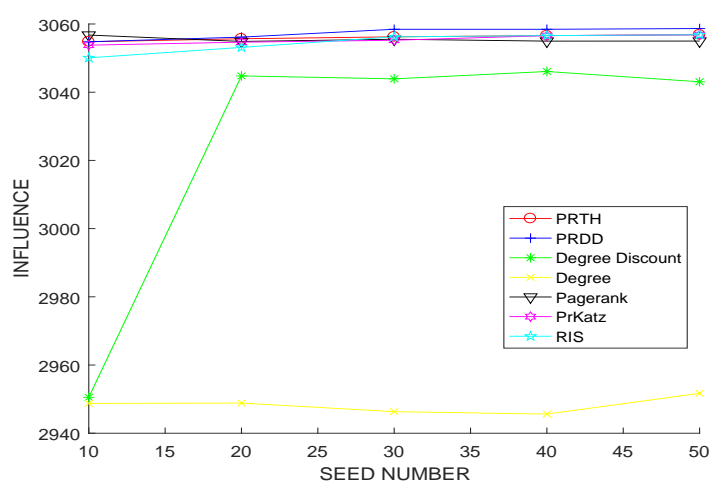

Fig. 3: The comparison on Facebook.

From Fig. 3, we can see that PRDD algorithm performs better in this dataset, while PageRank algorithm performs better merely at the beginning. Generally speaking, these four algorithms, including PageRank algorithm, PRTH algorithm, PrKatz algorithm and RIS algorithm, have similar effect. There is a gap between the Degree Discount algorithm and the above four algorithms, especially when $k$ is set to 10, the Degree algorithm performs the worst. In Facebook, RIS algorithm performs best, which shows that RIS algorithm is more suitable for the dataset with more edges. From Fig. 3, we can see that our algorithm is not much better than other algorithms for the dataset with large average degree.

Figure. 4 shows the effect of several algorithms in CAHepTh dataset. CA-HepTh has the minimum average degree compared with other three datasets.

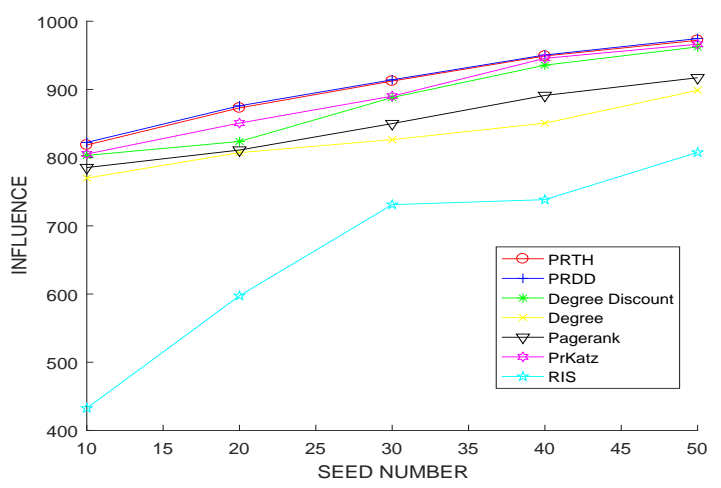

Fig. 4: The comparison on CA-HepTh.

From Fig. 4, we can see that the effect of PRTH algorithm and PRDD algorithm is similar, and the result of PRDD algorithm is slightly higher than that of PRTH algorithm. Also, 
we found that PrKatz algorithm achieved good results in this dataset, and the Degree Discount algorithm follows. At the same time, PageRank, Degree and RIS perform poorly. From this dataset, we can see that PRTH and PRDD have similar effect on relatively small-scale dataset.

Figure. 5 shows the influence diffusion comparison of our algorithms against with the baseline algorithms on NetHEPT. From Fig. 5, we can see that the PRTH algorithm is slightly

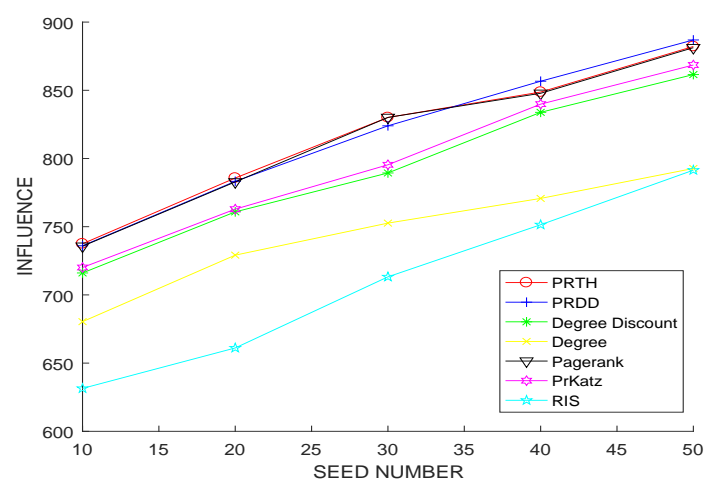

Fig. 5: The comparison on NetHEPT.

stronger than the original PageRank algorithm in the initial stage, and the latter two are almost the same. The reason is that there are many nodes with large pagerank value, which makes the PRTH algorithm not play a very important role. The two algorithms appear in the later period, and the growth slowly down, which is consistent with the conclusion that when the number of nodes becomes more, there will be point aggregation. For PRDD algorithm, we can intuitively see that its growth is relatively stable. In the early stage, the effect of PRDD algorithm is slightly worse than PRTH algorithm and original Pagerank algorithm. However, in the later stage, PRDD algorithm has been realized anti super, so we can see that the integration of degree discount and PRTH accomplish a better effect. At the same time, we can also conclude that the PRDD algorithm get a better improvement compared with the original Degree Discount algorithm. The overall effect of the PrKatz algorithm is similar to that of the Degree Discount algorithm. Moreover, we also find that RIS algorithm does not perform well in NetHEPT.

Figure. 6 shows the influence diffusion comparison of our algorithms against with the baseline algorithms on NetPHY.

From Fig. 6, we can find that the performance of the original Pagerank algorithm is poor, and the performance of PRTH algorithm works much better. It can be seen that PRTH algorithm plays a great role in NetPHY. But the disadvantage is that when $k$ is 30 , there is a relatively large fluctuation. We have already analyzed that the main disadvantage of PRTH is that it will be node aggregation. From the above results, we can see that there are more node aggregation at $k$ is 30. On the contrary, PRDD algorithm is more smoother and more effective than PRTH algorithm. Compared with PRDD, the original Degree Discount algorithm has a big gap. RIS

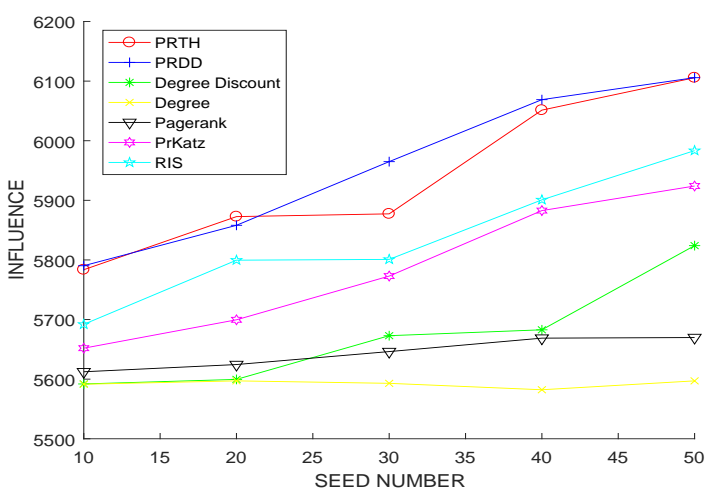

Fig. 6: The comparison on NetPHY.

algorithm is better than PrKatz algorithm in NetPHY.

Figure. 7 shows the running time of several algorithms on four datasets. Obviously, we can see that PrKatz algorithm runs the longest in four datasets. The reason is that it takes a long time to calculate the Katz centrality at the beginning. The second is RIS algorithm. RIS algorithm takes a long time because it needs to calculate the path. In all datasets, Degree algorithm gains the shortest time. Because Degree algorithm only needs to calculate the degree of each point and sort. Similarly, Pagerank algorithm only needs to calculate the Pagerank value of each point and sort. However, the calculation time of Pagerank is longer than that of degree, but it is much less than that of Katz centrality. So in all datasets, the time of Pagerank algorithm is the third shortest. As shown in Fig. 7, we can conclude that Degree Discount algorithm takes the second shortest time. Compared with Degree algorithm, Degree Discount algorithm needs much calculation cost. But its time-consuming is still shorter than Pagerank algorithm. We can know that there is a big gap between the calculation of Degree and Pagerank. Our algorithms, PRTH and PRDD, rank fourth and third in time-consuming, respectively. PRDD takes a longer time than PRTH, because PRDD combines PRTH and Degree Discount, so the time of PRDD is longer than the sum of PRTH and Degree Discount. Because PRTH algorithm is based on the calculation of Pagerank value, the time cost of it will be longer than Pagerank algorithm.

\section{CONCLUSION}

In this paper, we exploit two algorithms PRTH and PRDD to tackle IM problem. Our algorithms still provide acceptable results compared to other known methods, and make it possible to calculate the influence spread directly and accurately. Experimental results show the effectiveness of our model that can better influence diffusion. PRTH performs better in moderately degree datasets when there are fewer seed nodes in the network, while PRDD performs better when there are more seed nodes in the network.

\section{ACKNOWLEDGEMENTS}

This research is supported by the National Natural Science Foundation of China (Grant No. 61866029) and the Post- 


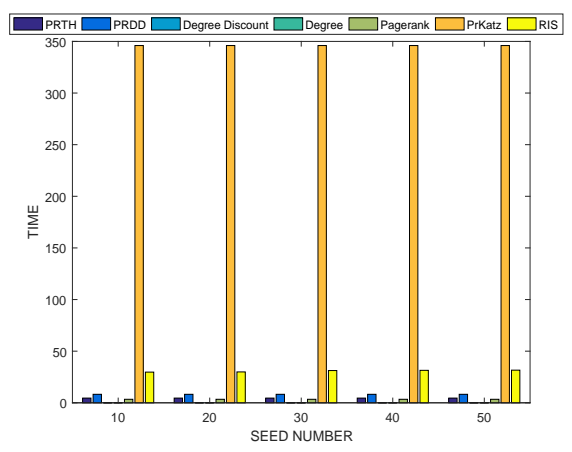

(a) Facebook

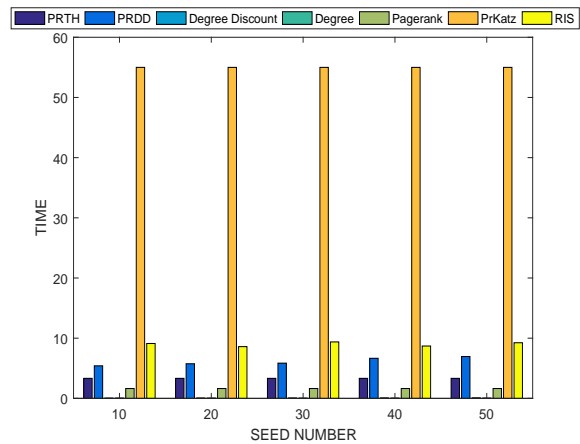

(c) NetHEPT.

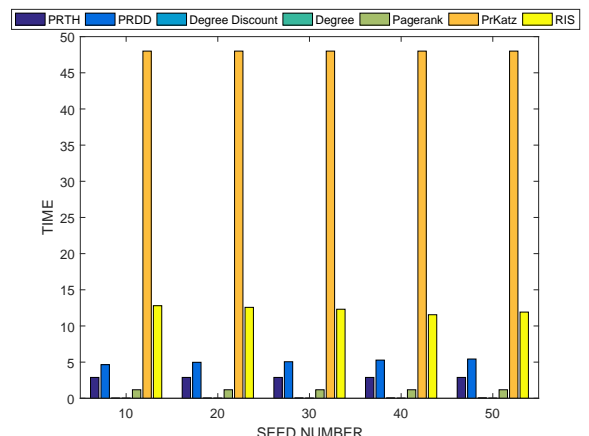

(b) CA-HepTh

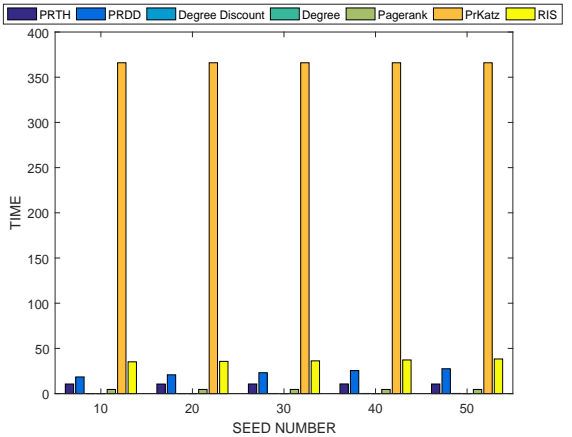

(d) NetPHY.

Fig. 7: Time comparison on four datasets.

graduate Innovation and Entrepreneurship Foundation of Inner Mongolia University (Grant No. 11200-121024).

\section{REFERENCES}

[1] M. Richardson and P. Domingos, "Mining knowledge-sharing sites for viral marketing," in Proceedings of the Eighth ACM SIGKDD International Conference on Knowledge Discovery and Data Mining, New York, NY, USA, jul 2002, pp. 61-70.

[2] D. Kempe, J. Kleinberg, and E. Tardos, "Maximizing the spread of influence through a social network," in Proceedings of the Ninth ACM SIGKDD International Conference on Knowledge Discovery and Data Mining, New York, NY, USA, aug 2003, pp. 137-146.

[3] J. Leskovec, A. Krause, C. Guestrin, C. Faloutsos, J. VanBriesen, and N. Glance, "Cost-effective outbreak detection in networks," in Proceedings of the 13th ACM SIGKDD International Conference on Knowledge Discovery and Data Mining, New York, NY, USA, aug 2007, pp. 420-429.

[4] A. Goyal, W. Lu, and L. V. Lakshmanan, "CELF ++: Optimizing the greedy algorithm for influence maximization in social networks," in Proceedings of the 20th International Conference Companion on World Wide Web, New York, NY, USA, mar 2011, pp. 47-48.

[5] W. Chen, Y. Wang, and S. Yang, "Efficient influence maximization in social networks," in Proceedings of the 15th ACM SIGKDD International Conference on Knowledge Discovery and Data Mining, New York, NY, USA, jun 2009, pp. 199-208.

[6] W. Chen, C. Wang, and Y. Wang, "Scalable influence maximization for prevalent viral marketing in large-scale social networks," in Proceedings of the 16th ACM SIGKDD International Conference on Knowledge Discovery and Data Mining, New York, NY, USA, jul 2010, pp. 1029_ 1038.

[7] G. Nandi, U. Sharma, and A. Das, "A novel hybrid approach for influence maximization in online social networks based on node neighborhoods," in Advances in Electronics, Communication and Computing, Singapore, oct 2018, pp. 509-520.

[8] X. Deng, Y. Dou, T. Lv, and Q. Nguyen, "A novel centrality cascading based edge parameter evaluation method for robust influence maximization," IEEE Access, vol. 5, pp. 22 119-22 131, oct 2017.
[9] S. M. Taheri, H. Mahyar, M. Firouzi, E. Ghalebi, R. Grosu, and A. Movaghar, "Hellrank: a hellinger-based centrality measure for bipartite social networks," Social Network Analysis and Mining, vol. 7, no. 22, may 2017.

[10] L. Cui, H. Hu, S. Yu, Q. Yan, Z. Ming, Z. Wen, and N. Lu, "Ddse: A novel evolutionary algorithm based on degree-descending search strategy for influence maximization in social networks," Journal of Network and Computer Applications, vol. 103, pp. 119-130, jul 2018.

[11] S. Lattanzi and Y. Singer, "The power of random neighbors in social networks," in Proceedings of the Eighth ACM International Conference on Web Search and Data Mining, New York, NY, USA, feb 2015, pp. 77-86.

[12] M. Alshahrani, Z. Fuxi, S. Mekouar, and S. Huang, "Top-k influential users selection based on combined katz centrality and propagation probability," in 2018 IEEE 3rd International Conference on Cloud Computing and Big Data Analysis (ICCCBDA), Chengdu, China, jun 2018, pp. 52-56.

[13] J. Ding, W. Sun, J. Wu, and Y. Guo, "Influence maximization based on the realistic independent cascade model," Knowledge-Based Systems, vol. 191, p. 105265, mar 2019.

[14] G. Maji, S. Mandal, and S. Sen, "A systematic survey on influential spreaders identification in complex networks with a focus on k-shell based techniques," Expert Systems with Applications, vol. 161, no. 113681 , dec 2020.

[15] S. Banerjee, M. Jenamani, and D. Pratihar, "Earned benefit maximization in social networks under budget constraint," Expert Systems with Applications, p. 114346, nov 2020.

[16] R. S. Wills, "Google's pagerank: The math behind the search engine," The Mathematical Intelligencer, vol. 28, no. 4, pp. 6-11, sep 2006.

[17] B. Christian, B. Michael, C. Jennifer, and L. Brendan, "Maximizing social influence in nearly optimal time," in Proceedings of the TwentyFifth Anпиal ACM-SIAM Symposium on Discrete Algorithms, USA, jan 2014, pp. 946-957. 